\title{
Detection of QTLs controlling fast kernel dehydration in maize (Zea mays L.)
}

Y.L. Qian ${ }^{1 *}$, X.Q. Zhang ${ }^{2 *}$, L.F. Wang ${ }^{3 *}$, J. Chen ${ }^{4}$, B.R. Chen ${ }^{5}$, G.H. Lv $^{6}$, Z.C. Wu ${ }^{7}$, J. Guo ${ }^{1}$, J. Wang ${ }^{1}$, Y.C. Qi ${ }^{1}$, T.C. Li ${ }^{1}$, W. Zhang ${ }^{1}$, L. Ruan ${ }^{1}$ and X.L. Zuo ${ }^{1}$

${ }^{1}$ The Laboratory of Maize Biotechnology, Tobacco Research Institute/Maize Research Center, Anhui Academy of Agricultural Science, Hefei, China ${ }^{2}$ The Laboratory of Crop Biotechnology, Crops Research Institute, Anhui Academy of Agricultural Sciences, Hefei, Anhui Province, China ${ }^{3}$ The Laboratory of Maize Biotechnology, Cereal Crops Institute, Henan Academy of Agricultural Sciences, Zhengzhou, China ${ }^{4}$ The Laboratory of Information Processing, Institute of Agricultural Economy and Information, Anhui Academy of Agricultural Science, Hefei, China ${ }^{5}$ The Laboratory of Crop Biotechnology, Institute of Crop Germplasm Resources, Jilin Academy of Agricultural Sciences, Gongzhuling, China

${ }^{6}$ The Laboratory of Crop Biotechnology, Dongyang Maize Research Institute of Zhejiang Province, Dongyang, China

${ }^{7}$ The Laboratory of Crop Biotechnology, Institute of Crop Science,

Chinese Academy of Agricultural Sciences, Beijing, China

*These authors contributed equally to this study.

Corresponding author: X.L. Zuo

E-mail:xlongzuo@yahoo.com

Genet. Mol. Res. 15 (3): gmr. 15038151

Received November 26, 2015

Accepted January 18, 2016

Published August 18, 2016

DOI http://dx.doi.org/10.4238/gmr.15038151

Copyright (C 2016 The Authors. This is an open-access article distributed under the terms of the Creative Commons Attribution ShareAlike (CC BY-SA) 4.0 License.

ABSTRACT. In order to understand the effect of grain moisture of inbred lines at the silking and physiological maturity stages on kernel 
dehydration rate, 59 maize inbred lines from six subgroups were selected. Grain moisture was measured and QTLs associated with kernel dehydration were mapped. A rapid dehydration evaluation and association analysis revealed eight inbred lines with faster dehydration rate, including Yuanwu 02, K36, Zhonger/O2, Lo1125, Han 49, Qi 319, Hua 160, and PH4CV. A single sequence repeat analysis using 85 pairs detected five QTLs with phenotypic variation contribution $\geq 10 \%$ in the permanent F2 generation populations Zheng $58 \times$ S1776 and Chang $7-2 \times$ K1131, which had LOD threshold values $\geq 3$ in both 2013 and 2014. The chromosome region of $q F k d r 7 b$ had not previously been reported and is preliminarily identified as a new major QTL. A false positive field verification of grain dehydration rate of 53 inbred lines indicated that the screening result of the rapid dehydration inbred lines by specific amplification with marker Phi114 was most similar to the field assessment result, followed by markers Phi127 and Phi029. The rapid dehydration lines selected based on primer Phi114 amplification were also similar to the field dehydration rate and can thus be used for molecular marker-assisted selection. A significant effort is needed to improve stress resistance and shorten the growth period via fast kernel dehydration in intermediate materials of the inbred lines K36, Zhonger/ O2, Lo1125, Han 49, Hua 160, and PH4CV, and further using the selected lines for new combinations.

Key words: Maize; Silking stage; Physiological maturity stage; QTLs; Fast kernel dehydration

\section{INTRODUCTION}

Maize (Zea mays L.) is a staple food for the majority of the world's population. It is also used as a key feed and industrial material. High quality maize is very competitive and has a broad market scope. In order to achieve a labor-saving production, mechanized harvesting is adopted and extended every day. However, high moisture kernels during the harvest may decrease maize quality, causing severe economic loss (Cao et al., 2013). After full ripening, the endosperm line shifts downward and disappears gradually, and a black kernel layer emerges when dry matter accumulation reaches the maximum, entering the physiological maturity stage. The dehydration rate at the physiological maturity stage determines kernel moisture and the maximum discrepancy in kernel moisture exists between different inbred lines and cultivars (Sala et al., 2006; Csiba et al., 2013). In 1910, Miller and Hughes, for the first time, reported that late maturing maize cultivars have a higher moisture content during harvest (Miller and Hughes, 1910). In the following 70 years, subsequent studies in the field progressed slowly, due to the complicated biological mechanism of maize kernel dehydration rate (Troyer and Ambrose, 1971; Hunter et al., 1979). With the development of biological science and technology, at the beginning of the 21 st century, great advances were made in physio-chemical mechanisms, inheritance, variation, and molecular mechanisms (Zhang et al., 2007; Reid et al., 2010; Frascaroli and Landi, 2013).

Rapid dehydration of physiologically mature maize kernels may reduce the moisture

Genetics and Molecular Research 15 (3): gmr.15038151 
content to a minimum, facilitating safe storage of maize (Ragai and Loomis, 1954). A number of studies have investigated the kernel dehydration rate of local hybrids (Cavalieri and Smith, 1985; Jin et al., 1997). However, the use of fast dehydration inbred lines in the production of maize has still not been implemented (Xiang et al., 2012). At present, a large number of studies have been conducted to identify and genetically analyze the dehydration rate of maize hybrids, and significant progress has been made. The maize grain dehydration rate at the late dough stage is influenced by external meteorological factors (Jin et al., 1997). A dehydration rate of maize varieties with grain moisture content equal to or higher than $30 \%$ following physiological maturity has been shown to be related to air temperature (Schmidt and Hallauer, 1966). Short husk and good peel permeability were shown to be conducive to rapid dehydration of maize grains (Crane et al., 1959). Rapid dehydration maize varieties exhibit high grain dehydration rates from physiological maturity to harvest and the dehydration rate was shown to be controlled mainly by additive genetic effects (Hallauer and Russell, 1962). The MIMYCS model for monitoring differences in grain moisture content between various maize varieties can be used to predict the occurrence of disease and the optimal prevention and harvest time (Maiorano et al., 2014).

There are many relevant results mentioned relating to the grain dehydration rate. For example, a heritable trait at 30-51 d post silking is shown (Zhang et al., 2007). From the perspective of QTLs, the study found 40 quantitative trait loci (QTLs) controlling for grain moisture content at four stages, using recombinant inbred lines between N04 and Dan 232 (Li et al., 2014). B73 x H99 test cross groups and detected QTLs controlling for grain moisture content were found on chromosomes 2 and 3 (Frascaroli et al., 2007). Six $F_{2: 3}$ groups and 11 mapped QTLs related to grain moisture content have been collected (Blanc et al., 2006). Thirteen QTLs in KW1265 x D146 test cross groups have been detected whose contribution rates to grain moisture content were above $10 \%$ (Melchinger et al., 1998). $\mathrm{BC}_{1} \mathrm{~F}_{1: 2}$ groups with FR1064 x GE440 were constructed and used to obtain five QTLs controlling for grain moisture content among which the effect values of a QTL on chromosome 6 reached $14 \%$ (Robertson-Hoyt et al., 2007. Among $\mathrm{F}_{3: 4}$ groups and 10 QTLs controlling for grain moisture content, the effect value of one QTL on chromosome 4 reached 20\% (Moreau et al., 2004). According to the comparative analysis with our study results, preliminary mapping results of $q F k d r 2 b$ and $q F k d r 2 d$ detected in our study were consistent with QTLs found by both Stuber et al. (1992) and Sala et al. (2006). The mapping results of $q F k d r 3 a$ and $q F k d r 3 c$ detected in our study also agreed with the QTLs found in previous studies (Ho et al., 2002; Capelle et al., 2010; Wang et al., 2012; Xiang et al., 2012).

Thus, screening of fast kernel dehydration inbred lines could be of great significance for maize production. In order to provide theoretical basis for screening of fast kernel dehydration cultivars, we conducted a measurement of grain moisture and mapped QTLs associated with kernel dehydration, using 59 maize inbred lines.

\section{MATERIAL AND METHODS}

\section{Experimental materials}

We used 59 shares of maize inbred lines, classified into six subgroups: PA, BSSS (Reid), Lyuda Red Cob (Lyuda Red Cob and its derivatives), PB, Lancaster, and Tangsipingtou (Tangsipingtou and its derivatives).

Genetics and Molecular Research 15 (3): gmr.15038151 


\section{Experimental design}

A randomized complete block design was adopted for the experimental design. Each plot contained a five m-long row with a spacing of $60 \mathrm{~cm}$ between rows and an inter-plant spacing of $25 \mathrm{~cm}$. The experiment was replicated thrice. In each plot, ten cobs were randomly selected and marked for measurement of cob moisture content at the silking and physiological maturity stages. For the cob moisture measurements, we used the inbred lines cultivated in the field at the Maize Research Centre, Anhui Academy of Agricultural Science at Ledong Li Autonomous County of Hainan Province in the winter 2013, and at Funan County of Anhui Province in the summer 2014.

\section{Measured items and methods}

The traditional drying method is not suitable for rapid detection of kernel moisture content. Instead, a moisture determination metric, which reveals kernel moisture via detection of electric capacity variation, has been developed (Reid et al., 2010). This method was listed in the International Seed Testing Protocol in 2003. In this study, an SK-300 moisture determination meter (The Japanese SANKU meter manufacturer, Tokyo, Honshu) was selected for the measurement.

\section{Data analysis}

All data were analyzed using Mapmaker/EXP 3.0 (Lincoln et al., 1993a), Mapmaker/ QTL 1.1 (Lincoln et al., 1993b) and Microsoft Excel 2007.

\section{RESULTS}

\section{Construction of a fast kernel dehydration evaluation method for maize inbred lines}

The moisture content discrepancy of inbred lines at the physiological maturity stage in Ledong in 2013 and Funan in 2014 was used to evaluate the fast dehydration character. If the mean of specific genotype was lower than the population mean, i.e., moisture content $\geq 0$, the inbred lines were regarded as fast dehydration inbred lines.

Ten shares of inbred lines were selected as fast dehydration inbred materials, accounting for $16.9 \%$ of the total material tested (Tables 1 and 2). Of these, the Yuanwu 02, K36, Zhonger/O2, Lo1125, Zao 49, Qi 319, Hua 160, and PH4CV lines assumed an evaluation score higher than 1 in both 2013 and 2014.

The analysis of the evaluation scores at different growth stages showed that the inbred lines with lower moisture at the physiological maturity stage are commonly fast dehydration materials, whereas those lacking a significant difference in moisture content between the silking and physiological maturity stages belong to the slow dehydration lines (Figures 1 and 2).

\section{Mapping QTLs associated with kernel dehydration rates in inbred lines}

In 2012, inducer powder from two F1 cross combinations, stock6 x Cauho inducer 1 and MT041 x KMS1105, was utilized to induce haploids with the two F1 cross combinations

Genetics and Molecular Research 15 (3): gmr.15038151 
Zheng $58 \times$ S1776 and Chang 7-2 x K1131. Until the putative haploid plants had grown to the 3-5-leaf stage, a doubling was performed by dripping a semi-lethal dose of colchicine $(1 \mathrm{~mL})$ at the base of each plant. A total of 237 DOUBLED HAPLOID F2 permanent Zheng 58 x S1776 plants and 275 DOUBLED HAPLOID F2 permanent Chang 7-2 x K1131 plants were obtained.

Table 1. Moisture content discrepancy scores for the silking and physiological maturity stages for the inbred lines with evaluation scores $\geq 1$ in the winter of 2013 , Hainan.

\begin{tabular}{l|l|c|c|c}
\hline No. & Line & Silking stage & Physiological maturity stage & Evaluation score \\
\hline 1 & Yuanwu 02 & -2.20 & 5.00 & 7.20 \\
\hline 2 & K36 & -2.11 & 2.78 & 4.88 \\
\hline 3 & Zhonger/O2 & -2.61 & 1.23 & 3.84 \\
\hline 4 & Lo1125 & -0.90 & 2.62 & 3.52 \\
\hline 5 & Zao 49 & -3.17 & 0.13 & 3.30 \\
\hline 6 & Qi 319 & -2.20 & -0.55 & 1.65 \\
\hline 7 & Hua 160 & 0.27 & 1.88 & 1.60 \\
\hline 8 & Yue 89E4-2 & -1.40 & 0.04 & 1.44 \\
\hline 9 & PH4CV & -1.13 & 0.19 & 1.32 \\
\hline
\end{tabular}

Table 2. Moisture content discrepancy scores for the silking and physiological maturity stages for the inbred lines with evaluation scores ${ }^{3} 1$ in the summer of 2014 , Anhui.

\begin{tabular}{l|l|c|c|c}
\hline No. & Line & Silking stage & Physiological maturity stage & Evaluation scores \\
\hline 1 & Yuanwu 02 & -2.42 & 4.93 & 7.35 \\
\hline 2 & K36 & -2.31 & 2.73 & 5.04 \\
\hline 3 & Zhonger/O2 & -2.86 & 1.20 & 4.07 \\
\hline 4 & Lo1125 & -0.98 & 2.57 & 3.56 \\
\hline 5 & Qi 319 & -2.43 & -0.56 & 1.87 \\
\hline 6 & Hua 160 & 0.30 & 1.84 & 1.54 \\
\hline 7 & Zao 49 & -1.33 & 0.11 & 1.44 \\
\hline 8 & PH4CV & -1.24 & 0.17 & 1.41 \\
\hline 9 & Ye 478 & -0.81 & 0.43 & 1.24 \\
\hline
\end{tabular}

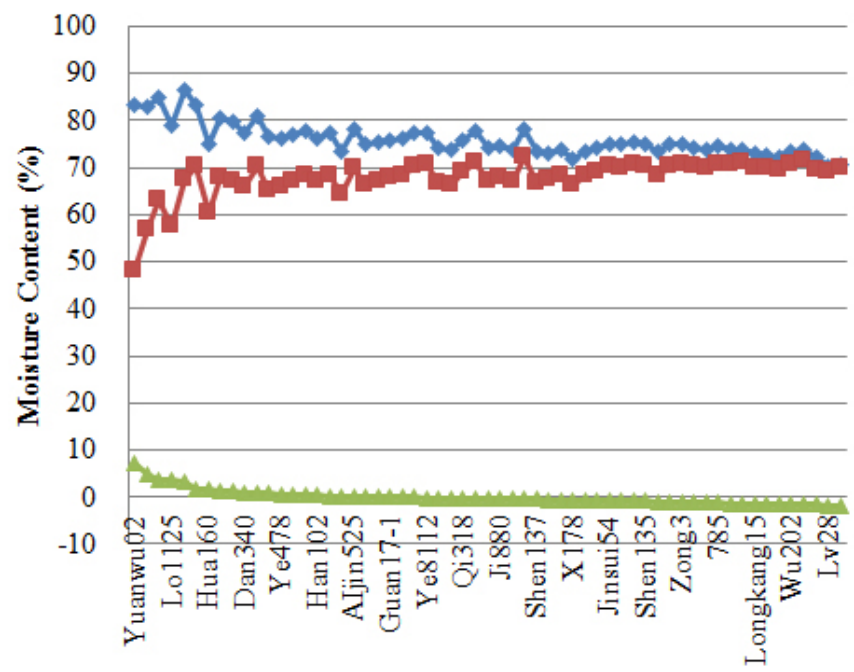

Figure 1. Moisture content discrepancy of 59 inbred lines at the silking (blue diamond) and physiological maturity (red squares) stages and the evaluation scores (green triangles) in the summer of 2013, Hainan. 


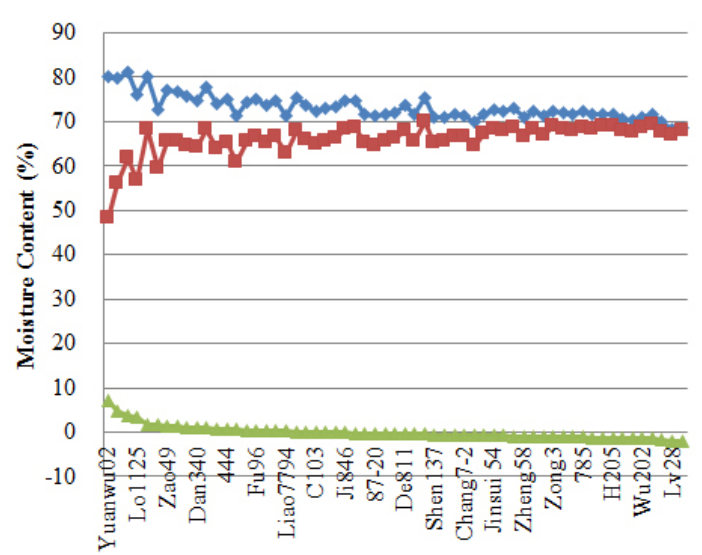

Figure 2. Moisture content discrepancy of 59 inbred lines at the silking (blue diamond) and physiological maturity (red squares) stages and the evaluation scores (green triangles) in the summer of 2014, Anhui.

In the summer of 2014, the kernel dehydration rates of these plants at the silking and physiological maturity stages were measured. Associated QTLs were detected for preliminary mapping, using 85 maize Simple Sequence Repeat (SSR) molecular marker published by the International Maize and Wheat Improvement Center ( 85 core SSR markers used in maize fingerprinting at $\mathrm{ABC}, \mathrm{CIMMYT}$, Mexico). Considering both the phenotypic observations and the SSR results, five QTLs with a phenotypic explanation rate $\geq 10 \%$ were obtained, using the LOD $\geq 3$ threshold. This provides useful information for the detailed mapping of QTLs associated with fast kernel dehydration (Figure 3).

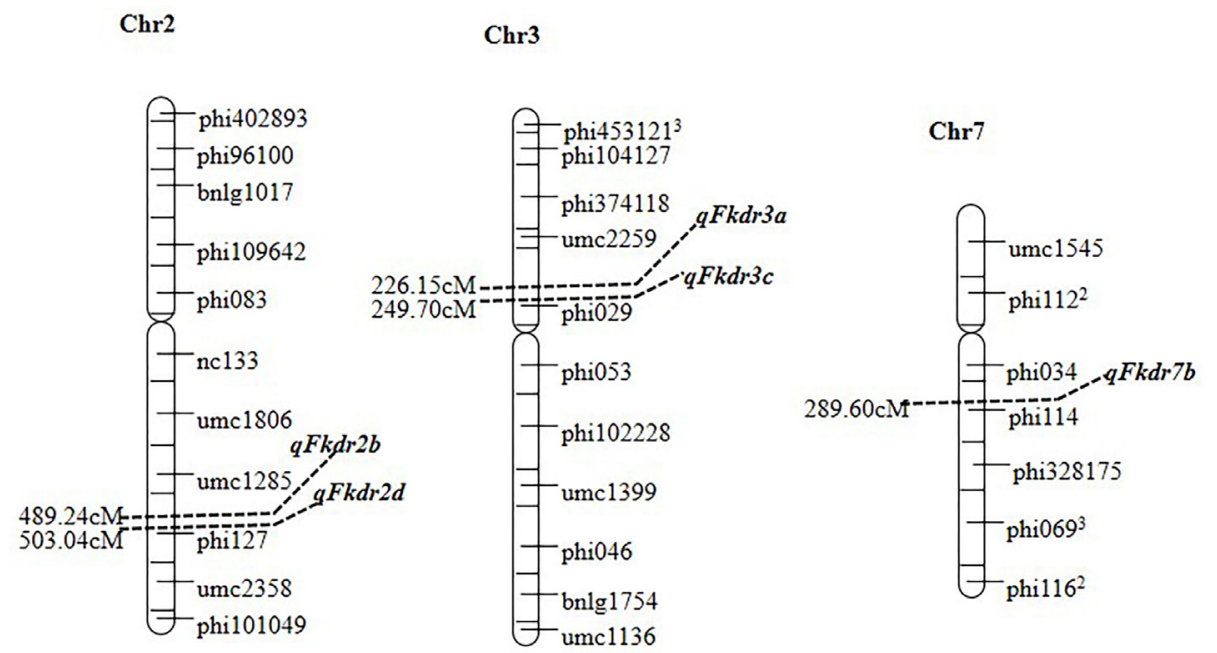

Figure 3. QTLs for maize fast kernel dehydration were detected by interval mapping. The presented LOD plots were obtained using the means of trait scores in 2013 and 2014. Linkage groups and the QTLs $q F k d r 2 b, q F k d r 3 a$, and $q F k d r 7 b$ from Zheng $58 \times$ S1776, $q F k d r 2 d$ and $q F k d r 3 c$ from Chang7-2 x K1131 designations are indicated. The significance threshold for interval mapping was determined at LOD $\geq 3$ and the distances in $\mathrm{cM}$ are indicated. 
QTLs controlling fast kernel dehydration in maize

\section{False positive testing of the linkage molecular marker}

False positive testing of the five identified QTLs was performed on 53 natural population shares. The specific fragment of primer Phi127 for the Zheng $58 \times$ S1776 and Chang 7-2 x K1131 populations could be amplified from the inbred lines Yuanwu 02, K36, Jiu 03, Fu 96, Guan 17-1, A632, Hua 160, PH4CV, 87-20, 444, Lu 65, Ji 880, Huobai, Ji 63, Huangzaosi, Huangyesi, CML67, 13A/O2, Yi 67, and 785. The specific fragment of primer Phi029 for the Zheng $58 \times$ S1776 and Chang 7-2 x K1131 populations could be amplified from the inbred lines Yuanwu 02, K36, Zhonger/O2, Lo1125, Zao 49, A632, Hua 160, and PH4CV. The fragment specific to primer Phil14 for the Zheng $58 \times$ S1776 population could be amplified from the inbred lines Yuanwu 02, K36, and Da MO. The screening result of rapid dehydration inbred lines exhibited the highest consistency with the field assessment result of dehydration rate using the specific amplification with marker Phi114, followed by markers Phi127 and Phi029.

\section{DISCUSSION}

In our study, the fast kernel dehydration lines have a lower moisture content at the physiological maturity stage. Because a discrepancy in growth period may affect the general evaluation of the samples, the population standard deviation was used to investigate the effect of growth period on kernel dehydration rate in our study. We found that the kernel begins to dehydrate prior to the physiological maturity stage and that the dehydration rate was not significantly affected by environmental factors at this stage in our study. Fast dehydration varieties share a high dehydration rate in physiologically mature kernels, and the heritability is approximately $77 \%$ (Brooking, 1990). When inbred lines with low moisture content at the physiological maturity stage from isolated populations were analyzed for their general and specific combining ability, the additive effect was found to contribute more than the non-additive effect when genetic composition had been selected (Sweeney et al., 1994). At days 30 and 51 post silking, kernel moisture content is not heritable, suggesting that kernel dehydration rate is not a heritable character (Zhang et al., 2007). It has also been reported that kernel moisture character can be passed to the progeny mainly in an additive manner (Xiang et al., 2012).

Based on an analysis of both domestic and foreign achievements, a large number of studies have studied maize grain dehydration rates. The grain dehydration rate of 142 maize elite inbred lines, local inbred lines, inbred lines improved during different periods, introduced lines, and high-generation breeding materials bred by the Maize Research Center of Anhui Academy of Agricultural Science, was determined in the field and eight rapid dehydration inbred lines were screened with the established grain dehydration assessment method (Xiang et al., 2012). From 2013 to 2014, permanent F2-generation doubled haploid groups were constructed, for the analysis of genetic diversity of 446 SSR primers and preliminary mapping of QTLs of rapid dehydration traits in maize grains. According to the comparative analysis, preliminary mapping results of $q F k d r 2 b$ and $q F k d r 2 d$ detected in our study were consistent with QTLs found by both Stuber et al. (1992) and Sala et al. (2006). The mapping results of $q F k d r 3 a$ and $q F k d r 3 c$ detected in our study also agreed with the QTLs found in previous studies (Ho et al., 2002; Capelle et al., 2010; Wang et al., 2012; Xiang et al., 2012). However, the chromosome region of $q F k d r 7 b$, which was preliminarily identified as a new major QTL, had not yet been reported. The false positive field verification of grain dehydration rate of

Genetics and Molecular Research 15 (3): gmr.15038151 
the 53 inbred lines showed that the screening result of rapid dehydration inbred lines by specific amplification using marker Phi114 exhibited the highest consistency with the field assessment of dehydration rate, followed by markers Phi127 and Phi029. In this study, using Zheng58 x S1776 doubled haploid groups, from the genetics and molecular biology perspective, genetic analyses of rapid dehydration traits in S1776 inbred lines were performed. Rapid dehydration genes were then mapped, deepening our theoretical understanding for the genetic mechanism of rapid dehydration of maize grains. In future studies, several molecular markers suitable for breeding rapid dehydration maize varieties could be developed, with the aim to promote the application and development of rapid dehydration maize inbred lines and enriching the basic theoretical system for rapid dehydration of maize inbred lines.

In this study, eight inbred fast kernel dehydration lines, including Yuanwu 02, K36, Zhonger/O2, Lo1125, Zao 49, Qi 319, Hua 160, and PH4CV were selected, considering both the fast kernel dehydration and the associated analysis. To build on the fast dehydration character, the inbred lines used here can be used for cross testing. The specific amplification of primer Phi029 is in accordance with field phenotypic observation results and can thus be used for marker-assisted selection. Primers Phi127 and Phi114 may be useful for finescale mapping of QTLs associated with the fast dehydration character, for further functional analysis of candidate genes. Under the high temperature and high humidity conditions in the North China Plain summer, the selected lines K36, Zhonger/O2, Lo1125, Zao 49, Hua 160, and $\mathrm{PH} 4 \mathrm{CV}$ can be used as intermediate materials for improving resistance and shortening the growth period. Use of the inbred lines with the fast kernel dehydration character for cross testing can also improve kernel appearance and color, further enhancing the apparent quality and economic benefits of the maize kernels. Moreover, it can decrease the kernel moisture content to within the safe preservation range quickly, in a labor- and cost-saving manner. To conclude, selection of maize resources and parental materials with fast kernel dehydration characters is in accordance with the mechanized harvesting trend in modern agriculture and is a very promising prospect.

\section{Conflicts of interest}

The authors declare no conflict of interest.

\section{ACKNOWLEDGMENTS}

We are grateful for funding received from the Creative Foundation of Anhui Agricultural Academy of Sciences (\#16B0916), the Seed Project Program of Anhui Agricultural Academy of Sciences (\#16D0911), the Scientific and Technological Innovation Team of the Anhui Academy of Agricultural Science (\#15C902), the Creative Foundation of Anhui Agricultural Academy of Sciences (\#14B0946), the Discipline Construction Program of Anhui Agricultural Academy of Sciences (\#14A0925), and the Key Science and Technology Research and Development Program of Anhui Province (\#1604a0702021).

Genetics and Molecular Research 15 (3): gmr.15038151 


\section{REFERENCES}

Blanc G, Charcosset A, Mangin B, Gallais A, et al. (2006). Connected populations for detecting quantitative trait loci and testing for epistasis: an application in maize. Theor. Appl. Genet. 113: 206-224.http://dx.doi.org/10.1007/s00122006-0287-1

Brooking IR (1990). Maize ear moisture during grain-filling, and its relation to physiological maturity and grain-drying. Field Crops Res. 23: 55-68. http://dx.doi.org/10.1016/0378-4290(90)90097-U

Cao A, Santiago R, Ramos AJ, Marín S, et al. (2013). Environmental factors related to fungal infection and fumonisin accumulation during the development and drying of white maize kernels. Int. J. Food Microbiol. 164: 15-22. http:// dx.doi.org/10.1016/j.ijfoodmicro.2013.03.012

Capelle V, Remoué C, Moreau L, Reyss A, et al. (2010). QTLs and candidate genes for desiccation and abscisic acid content in maize kernels. BMC Plant Biol. 10: 2. http://dx.doi.org/10.1186/1471-2229-10-2

Cavalieri AJ and Smith OS (1985). Grain filling and field drying of a set of maize hybrids released from 1930 to 1982. Crop Sci. 25: 856-860. http://dx.doi.org/10.2135/cropsci1985.0011183X002500050031x

Crane PL, Miles SR and Newman JE (1959). Factors associated with varietal differences in rate of field drying in corn. Agron. J. 51: 318-320. http://dx.doi.org/10.2134/agronj1959.00021962005100060003x

Csiba M, Kovács AJ, Virág I and Neményia M (2013). The most common errors of capacitance grain moisture sensors: effect of volume change during harvest. Precis. Agric. 14: 215-223. http://dx.doi.org/10.1007/s11119-012-9289-y

Frascaroli E and Landi P (2013). Divergent selection in a maize population for germination at low temperature in controlled environment: study of the direct response, of the trait inheritance and of correlated responses in the field. Theor. Appl. Genet. 126: 733-746.http://dx.doi.org/10.1007/s00122-012-2014-4

Frascaroli E, Canè MA, Landi P, Pea G, et al. (2007). Classical genetic and quantitative trait loci analyses of heterosis in a maize hybrid between two elite inbred lines. Genetics 176: 625-644. http://dx.doi.org/10.1534/genetics.106.064493

Hallauer AR and Russell WA (1962). Estimates of maturity and its in heritance in maize. Crop Sci. 2: 289-295. http:// dx.doi.org/10.2135/cropsci1962.0011183X000200040006x

Ho JC, McCouch SR and Smith ME (2002). Improvement of hybrid yield by advanced backcross QTL analysis in elite maize. Theor. Appl. Genet. 105: 440-448. http://dx.doi.org/10.1007/s00122-002-0945-x

Hunter RB, Mortimore G, Gerrish EE and Kannenberg LW (1979). Field drying of flint and dent endosperm maize. Crop Sci. 19: 401-402. http://dx.doi.org/10.2135/cropsci1979.0011183X001900030031x

Jin Y, Wang ZH, Zhang YL, Wang SH, et al. (1997). Difference analysis on the natural dry rate of kernel after wax ripening in maize hybrids. J. Northeast Agric. Univ. 28: 29-32.

Li YL, Dong YB, Yang ML, Wang QL, et al. (2014). QTL detection for grain water relations and genetic correlations with grain matter accumulation at four stages after pollination in maize. Plant Biochem. Physiol 2: 1-9.

Lincoln SE, Daly MJ and Lander ES (1993a). Constructing genetic linkage maps with MAPMAKER/EXP version 3.0. A Whitehead Institute for Bio-medical Research Technical Report, 3rd edn.

Lincoln SE, Daly MJ and Lander ES (1993b). Mapping genes controlling quantitative traits using MAPMAKER/QTL version 1.1. A Whitehead Institute for Bio-medical Research Technical Report, 2nd edn.

Maiorano A, Fanchini D and Donatelli M (2014). MIMYCS.Moisture, a process-based model of moisture content in developing maize kernels. Eur. J. Agron. 59: 86-95. http://dx.doi.org/10.1016/j.eja.2014.05.011

Melchinger AE, Utz HF and Schön CC (1998). Quantitative trait locus (QTL) mapping using different testers and independent population samples in maize reveals low power of QTL detection and large bias in estimates of QTL effects. Genetics 149: 383-403.

Miller MF and Hughes HD (1910). Cooperative variety tests of corn, variety tests of corn at Columbia. Missouri Agric. Exp. Stn. Bull 87: 97-158.

Moreau L, Charcosset A and Gallais A (2004). Use of trial clustering to study QTL x environment effects for grain yield and related traits in maize. Theor. Appl. Genet. 110: 92-105. http://dx.doi.org/10.1007/s00122-004-1781-y

Ragai H and Loomis WE (1954). Respiration of maize grain. Plant Physiol. 29: 49-55.http://dx.doi.org/10.1104/pp.29.1.49

Reid LM, Zhu X, Morrison MJ, Woldemariam T, et al. (2010). A non-destructive method for measuring maize kernel moisture in a breeding program. Maydica 55: 163-171.

Robertson-Hoyt LA, Kleinschmidt CE, White DG, Payne GA, et al. (2007). Relationships of resistance to Fusarium ear rot and fumonisin contamination with agronomic performance of maize. Crop Sci. 47: 1770-1778. http://dx.doi. org/10.2135/cropsci2006.10.0676

Sala RG, Andrade FH, Camadro EL and Cerono JC (2006). Quantitative trait loci for grain moisture at harvest and field grain drying rate in maize (Zea mays, L.). Theor. Appl. Genet. 112: 462-471. http://dx.doi.org/10.1007/s00122-005-0146-5

Schmidt JL and Hallauer AR (1966). Estimating harvest date of corn in the field. Crop Sci. 6: 227-231. http://dx.doi. org/10.2135/cropsci1966.0011183X000600030003x

Genetics and Molecular Research 15 (3): gmr.15038151 
Stuber CW, Lincoln SE, Wolff DW, Helentjaris T, et al. (1992). Identification of genetic factors contributing to heterosis in a hybrid from two elite maize inbred lines using molecular markers. Genetics 132: 823-839.

Sweeney PM, St. Martin SK and Clucas CP (1994). Indirect inbred selection to reduce grain moisture in maize hybrids. Crop Sci. 34: 391-396. http://dx.doi.org/10.2135/cropsci1994.0011183X003400020016x

Troyer AF and Ambrose WB (1971). Plant characteristics affecting field drying rate of ear corn. Crop Sci. 11: 529-531. http://dx.doi.org/10.2135/cropsci1971.0011183X001100040019x

Wang ZH, Wang X, Zhang L, Liu XJ, et al. (2012). QTL underlying ðeld grain drying rate after physiological maturity in maize (Zea mays L.). Euphytica 185: 521-528. http://dx.doi.org/10.1007/s10681-012-0676-2

Xiang K, Reid LM, Hang ZM, Zhu XY, et al. (2012). Characterization of correlation between grain moisture and ear rot resistance in maize by QTL meta-analysis. Euphytica 183: 185-195. http://dx.doi.org/10.1007/s10681-011-0440-z

Zhang F, Wan XQ and Pan GT (2007). Molecular mapping of QTL for resistance to maize ear rot caused by Fusarium moniliforme. Acta Agron. Sin. 33: 491-496.

Genetics and Molecular Research 15 (3): gmr.15038151 